\title{
Disseminated Mucormycosis in a Patient with Recent Kidney Transplantation: A Case Report and Review of the Literature
}

\author{
Mohamad Hazem Hatahet Mohanram Narayanan Constance Cleaves \\ Riyam Zreik
}

Scott \& White Healthcare/Texas A\&M Health Science Center College of Medicine, Temple, Tex., USA

\section{Key Words}

Mucormycosis $\cdot$ Kidney transplant $\cdot$ Immunosuppression

\begin{abstract}
Mucormycosis is an invasive fungal infection commonly seen in diabetics and immunocompromised patients. We report a case of disseminated mucormycosis in a 47-year-old diabetic male who underwent deceased donor renal allograft transplantation about 5 weeks prior to presentation. Our patient presented with increasing fatigue, diarrhea and oligoanuria and was found to have significant acute kidney injury. Doppler ultrasound of the allograft revealed segmental decreased renal perfusion in the upper pole of the allograft with moderate hydronephrosis. Nephrostomy tube placement yielded minimal urine output. An allograft biopsy showed diffuse C4d-positive staining and fungal hyphae suggestive of Mucor infection. Computed tomography (CT) imaging revealed a right upper lobe mass, a small hypodensity in the liver and normal findings in the head. Despite prompt management including discontinuation of immunosuppression, amphotericin B and allograft nephrectomy, the patient had a rapid decompensation, developed respiratory failure requiring intubation, hypotension and supraventricular tachycardia with multiple new areas of hypoattenuation on head CT - all of which ultimately resulted in his death. A review of the literature revealed that mucormycosis is a relatively rare disease with a cumulative 12 -month incidence rate of $0.07 \%$ in solid organ transplant recipients. Disseminated disease was found in about $23 \%$ of cases, with a mortality rate of $96 \%$.
\end{abstract}




\section{Introduction}

Mucormycosis is an aggressive, life-threatening infection usually associated with devastating outcomes. It is caused by Mucorales molds that are ubiquitous spore-forming organisms found in the soil. The infection usually affects immunocompromised patients, especially those with diabetes mellitus. It has a relatively rare incidence among solid organ transplant (SOT) recipients. The organism is angioinvasive and known to cause infarction of infected tissues. When the infection becomes disseminated, the disease caries a mortality rate greater than $95 \%$.

\section{Case}

A 47-year-old Hispanic man with a history of end-stage kidney disease secondary to diabetic nephropathy underwent deceased donor renal allograft transplantation. His immediate posttransplant course was unremarkable, with prompt functioning of his allograft and decreasing creatinine levels. His immunosuppressant regimen included tacrolimus, mycophenolate sodium and corticosteroids. Ten days after the transplant, he developed suprapubic abdominal pain that was thought to be due to the indwelling ureteral stent, necessitating its removal with complete resolution of his discomfort.

Approximately 5 weeks following his transplant, the patient presented with fatigue, diarrhea and oligoanuria, without any associated fever or respiratory symptoms. He had significant allograft dysfunction with a creatinine level of $8.1 \mathrm{mg} / \mathrm{dl}$ up from a nadir of 2.7 $\mathrm{mg} / \mathrm{dl}$. Doppler ultrasound of the renal allograft showed an area of hypoperfusion affecting the upper pole of the renal allograft, with a moderate degree of hydronephrosis. A percutaneous nephrostomy tube was placed without any improvement in urine output or renal function. A renal allograft biopsy was then done to further elucidate the etiology of his graft dysfunction. This revealed diffuse C4d-positive staining in the peritubular capillaries suggestive of acute humoral rejection; however, the biopsy was also remarkable for numerous, broad, ribbon-like, nonseptate, fungal hyphae with wide-angle branching suggestive of Mucor infection. There was a focal cortical infarct accounting for approximately $10 \%$ of the overall biopsy, with focal small vessels containing fungal hyphae (fig. 1). The patient was prepared for transplant nephrectomy and arteriovenous fistula thrombectomy. Postoperatively, his anti-rejection medications were discontinued, amphotericin B and micafungin were started and hemodialysis was initiated.

A pathology exam performed after the nephrectomy (fig. 2a, b) showed significant cortical infarction involving more than half of the cortex, with patchy, widespread involvement of fungal hyphae that involved the ureteral lumen, ureteral wall, renal parenchyma and numerous large and small vessels. Chest CT showed a large right upper lobe cavitating mass measuring $4.9 \times 2.8 \mathrm{~cm}$, with scattered areas of smaller nodules present throughout both lungs (fig. 3). Abdominal CT revealed a wedge-shaped area of hypodensity within the anterior spleen compatible with infarction, along with additional areas of hypodensity within the spleen. There was also a nonspecific hypodensity in the right hepatic lobe. Head and maxillofacial CT were normal. The patient underwent a CT-guided biopsy of the right upper lobe of the lung, and pathology confirmed the clinical suspicion of disseminated mucormycosis. Within 1 week of presentation, the patient became hemodynamically unstable, requiring a transfer to the intensive care unit for mechanical ventilation and vasopressor support. As his mental status showed significant decline, a repeat head CT was done. This revealed interval development of areas of subcortical hypoattenuation involving 
the bilateral frontal lobes, bilateral occipital lobes and bilateral cerebral hemispheres, suggesting multifocal cerebritis with no abscesses (fig. 4). The patient's condition continued to deteriorate, and he developed supraventricular tachycardia and the inability to tolerate hemodialysis. His family elected for comfort care and the patient subsequently died.

The patient's records did not reveal any previous history of significant upper or lower respiratory symptoms, and his previous chest X-rays, including the one that was done just before his transplant surgery, were unremarkable. A renal allograft biopsy was done on the recipient of the donor-matched organ (the second donor kidney) and showed no evidence of fungal infection, which made it unlikely to be a donor-transmitted infection.

\section{Discussion}

Infection with Mucorales is a serious condition in immunocompetent individuals, with innate immunity generally being sufficient to protect against this infection [1]. SOT recipients are at risk for mucormycosis. Other risk factors for mucormycosis include diabetes mellitus, hematopoietic stem cell transplantation, certain malignancies [2], glucocorticoid treatment [3] and iron overload [4]. Our patient had several of these risk factors, including long-standing diabetes mellitus, kidney transplant recipient status and glucocorticoid treatment as part of his anti-rejection medication regimen.

The infected tissues usually develop infarction and necrosis as a result of blood vessel invasion by hyphae. Incidence has been on the rise over the past few decades but is variable in SOT recipients, ranging from 0.4 to $16.0 \%$ depending on the reference [1], with an average of $1.1 \%$ in kidney transplant recipients [5]. Park et al. [6] found that the 12-month cumulative incidence of mucormycosis was $0.07 \%$ in SOT recipients in the multicenter TRANSNET study. This study also identified geographic differences within the USA, with $46 \%$ of cases occurring in the South compared with 35, 17 and 7\% in the Midwest, West and Northeast, respectively.

The onset of mucormycosis after kidney transplant varied between 1 month and 4 years [7]; in our case, the onset was about 5 weeks after his transplant. The onset was found to occur earlier in liver transplant recipients (median, 0.8 months after transplant) compared to SOT recipients (median, 5.7 months) [8]. One study reported surgical wound infection a few days after kidney transplant [9]. Disease presentation depends on the affected organs, with rhino-orbital-cerebral infection being the most common presentation seen in $34 \%$ of cases, followed by pulmonary in $26 \%$, cutaneous in $17 \%$, gastrointestinal in $12 \%$ and renal in $6 \%$. Disseminated disease was found in $14 \%$ of cases [10].

Diagnosis depends on finding the fungal hyphae by pathologic exam of infected tissues. The morphologic features include broad, ribbon-like, thin-walled, nonseptate hyphae with wide-angle branching. Samples from suspected cases often fail to grow Mucorales in microbiologic cultures. Currently, there are no available serological tests for routine diagnostics, and the use of polymerase chain reaction (PCR) has been recently reported. In 1 study, culture was positive in $44 \%$ of pathologically proven cases, while PCR was positive in $81 \%$ [11]. This correlates very well with our case, in which the diagnosis was made by the characteristic pathologic findings in the absence of positive PCR and fungal cultures.

Treatment usually includes aggressive debridement of infected and necrotic tissues, antifungal agents and withdrawal of immunosuppressive drugs. Continuation of treatment is recommended until full resolution of the infection is achieved, although this usually takes several months. Lipid formulations of amphotericin B have evolved as the cornerstone of primary therapy for mucormycosis. Posaconazole may be useful as salvage therapy, and 
Hatahet et al.: Disseminated Mucormycosis in a Patient with Recent Kidney Transplantation: A Case Report and Review of the Literature

combination therapy has been recommended to improve outcomes [12]. Adding the iron chelator deferasirox has also shown better response in animal models [13], while delaying antifungal treatment has been associated with a significant increase in mortality [14].

The prognosis for mucormycosis is very poor even with aggressive treatment. Roden et al. [15] reviewed 929 mucormycosis cases and found that mortality varied with infection site. The infection was fatal in $96 \%$ of patients with disseminated disease, in $85 \%$ with gastrointestinal infection and in $76 \%$ with pulmonary infection. Disseminated infection developed in $23 \%$ of cases.

\section{References}

1 Lanternier F, Sun HY, Ribaud P, Singh N, Kontoyiannis DP, Lortholary O: Mucormycosis in organ and stem cell transplant recipients. Clin Infect Dis 2012;54:1629-1636.

-2 Kontoyiannis DP, Lionakis MS, Lewis RE, Chamilos G, Healy M, Perego C, Safdar A, et al: Zygomycosis in a tertiary-care cancer center in the era of Aspergillus-active antifungal therapy: case-control observational study of 27 recent cases. J Infect Dis 2005;191:1350-1360.

3 Kontoyiannis DP, Wessel VC, Bodey GP, Rolston KV, et al: Zygomycosis in the 1990s in a tertiary-care cancer center. Clin Infect Dis 2000;30:851-856.

-4 Maertens J, Demuynck H, Verbeken EK, Zachee P, Verhoef GE, Vandenberghe P, et al: Mucormycosis in allogeneic bone marrow transplant recipients: report of five cases and review of the role of iron overload in the pathogenesis. Bone Marrow Transplant 1999;24:307-312.

-5 Nampoory MR, Khan ZU, Johny KV, Constandi JN, Gupta RK, Al-Muzairi I, et al: Invasive fungal infections in renal transplant recipients. J Infect 1996;33:95-101.

6 Park BJ, Pappas PG, Wannemuehler KA, Alexander BD, Anaissie EJ, Andes DR, et al: Invasive non-Aspergillus mold infections in transplant recipients, United States, 2001-2006. Emerg Infect Dis 2011;17:1855-1864.

7 Aslani J, Eizadi M, Kardavani B, Khoddami-Vishteh HR, Nemati E, Hoseini SM, et al: Mucormycosis after kidney transplantations: report of seven cases. Scand J Infect Dis 2007;39:703-706.

-8 Singh N, Aguado JM, Bonnatti H, Forrest G, Gupta KL, Safdar N, et al: Zygomycosis in solid organ transplant recipients: a prospective, matched case-control study to assess risks for disease and outcome. J Infect Dis 2009;200:1002-1011.

-9 Zhao L, Wang CX, Zhang L, Tu XA, Wang W, Chen Y, et al: Mucormycosis extending from the surgical wound to the transplanted kidney: case report and literature review. Exp Clin Transplant 2012;10:403-405.

10 Almyroudis NG, Sutton DA, Linden P, Rindaldi MG, Fung J, Kusne S: Zygomycosis in solid organ transplant recipients in a tertiary transplant center and review of the literature. Am J Transplant 2006;6:2365-2374

11 Hammond SP, Bialek R, Milner DA, Petchnigg EM, Baden LR, Marty FM: Molecular methods to improve diagnosis and identification of mucormycosis. J Clin Microbiol 2011;49:2151-2153.

-12 Kontoyiannis DP, Lewis RE: How I treat mucormycosis. Blood 2011;118:1216-1224.

-13 Spellberg B, Walsh TJ, Kontoyiannis DOP, Edwards J Jr, Ibrahim AS: Recent advances in the management of mucormycosis: from bench to bedside. Clin Infect Dis 2009;48:1743.

14 Chamilos G, Lewis RE, Kontoyiannis DP: Delaying amphotericin B-based frontline therapy significantly increases mortality among patients with hematologic malignancy who have zygomycosis. Clin Infect Dis 2008;47:503-509.

15 Roden MM, Zaoutis TE, Buchanan WL, Knudsen TA, Sarkiisova TA, Schaufele RL, et al: Epidemiology and outcome of zygomycosis: a review of 929 reported cases. Clin Infect Dis 2005;41:634-653. 


\section{Case Reports in \\ Nephrology and Urology}

Case Rep Nephrol Urol 2013;3:58-63

DOI: $10.1159 / 000351517$

atahet et al: Disseminated Mucormycosis in a Patient with Recent Kidney

Transplantation: A Case Report and Review of the Literature

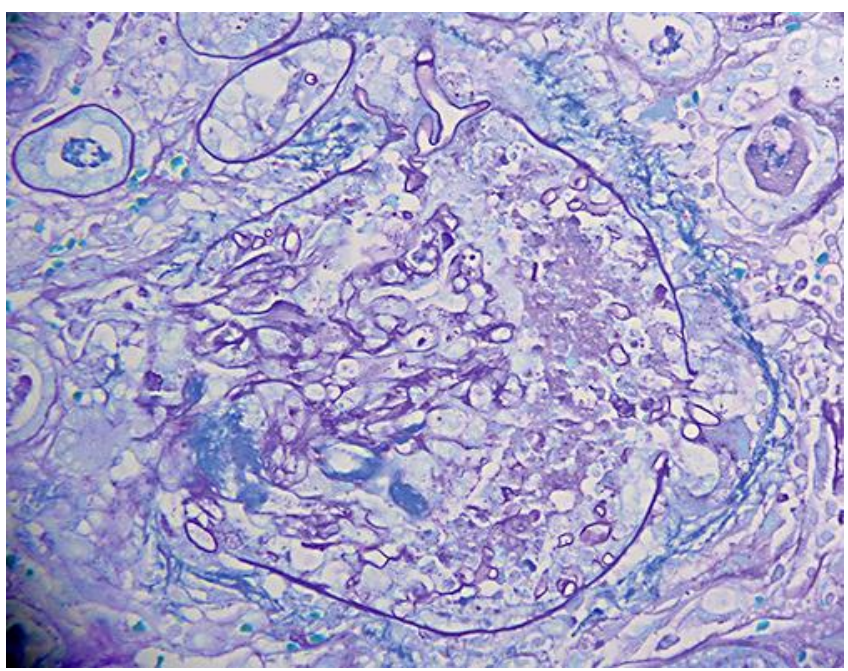

Fig. 1. High-power PAS stain shows a glomerulus infiltrated by fungal hyphae. $\times 40$.
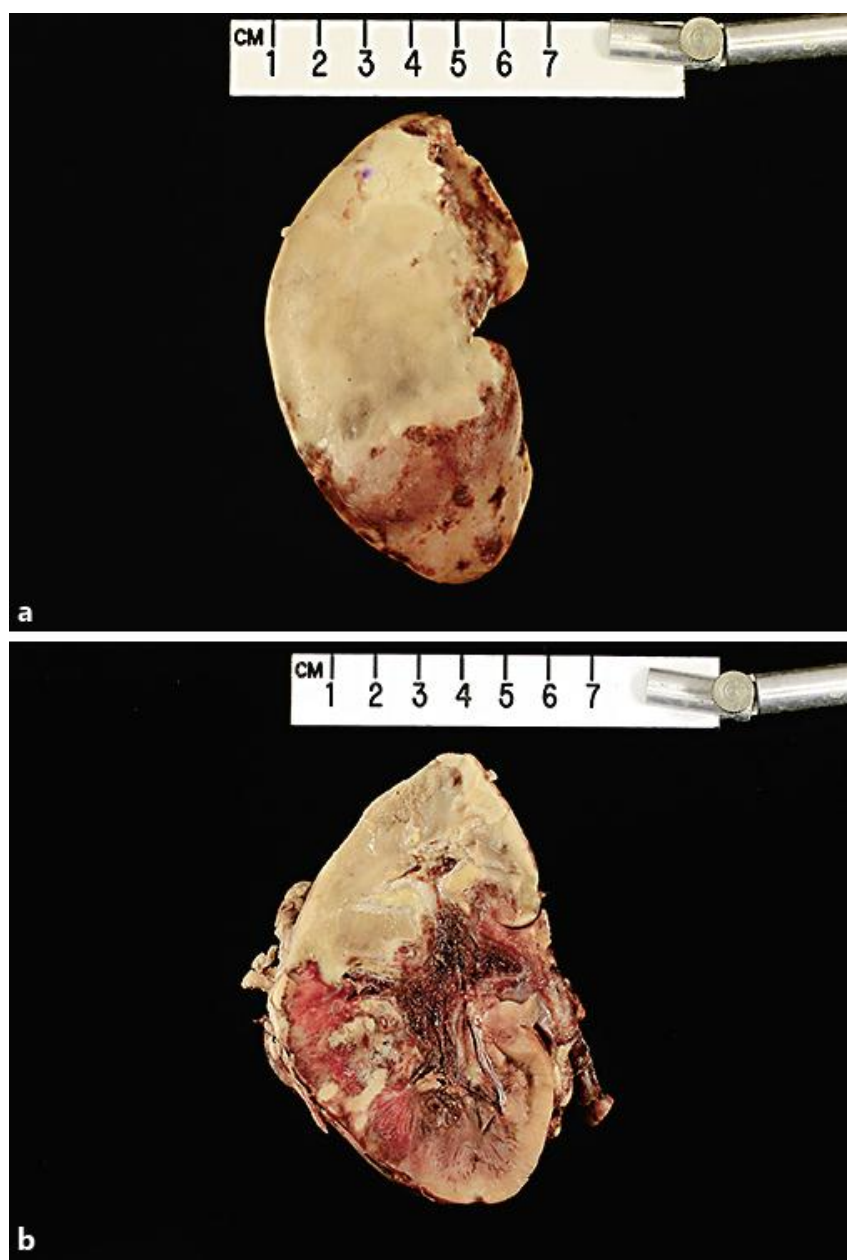

Fig. 2. a Gross pathology specimen with a large cortical infarct. b Cut specimen with a large cortical infarct. 
Case Reports in

Nephrology and

Urology
Case Rep Nephrol Urol 2013;3:58-63

DOI: $10.1159 / 000351517$

Thatahet et al: Disseminated Mucormycosis in a Patient with Recent Kidney

Transplantation: A Case Report and Review of the Literature

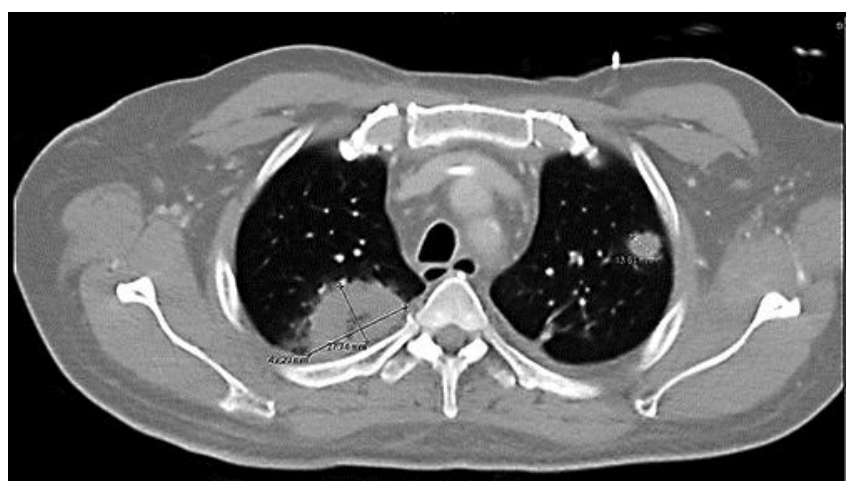

Fig. 3. Chest CT shows a $4.9 \times 2.8$-cm mass with cavitation within the posterior aspect of the right upper lobe and a moderate-sized area of consolidation involving the left lower lobe.

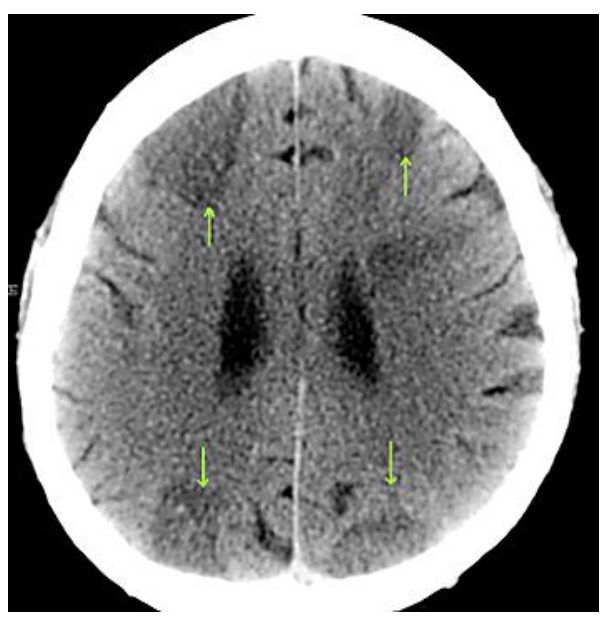

Fig. 4. Head CT reveals cortical hypoattenuation (arrows) within the bilateral frontal and occipital lobes with no peripheral enhancing. 